\title{
Nonalcoholic Fatty Liver Disease Associated with Obstructive Sleep Apnea: Just a Coincidence?
}

\author{
Carla Daltro • Helma P. Cotrim • Erivaldo Alves • \\ Luiz Antônio de Freitas • Leila Araújo • \\ Leonardo Boente • Rafael Leal • Thaís Portugal
}

Published online: 17 June 2010

(C) Springer Science+Business Media, LLC 2010

\begin{abstract}
Background Obesity is associated with obstructive sleep apnea (OSA) and nonalcoholic fatty liver disease (NAFLD). It has been shown that OSA could be an independent risk factor for NAFLD. OSA could cause not only insulin resistance but worse NAFLD through nocturnal hypoxemia. This study aimed to evaluate the frequency of OSA and NAFLD in obese patients and the relationship between OSA, insulin resistance, and severity of steatohepatitis (nonalcoholic steatohepatitis (NASH)).

Methods Forty obese patients submitted to bariatric surgery were evaluated. Sleep studies, fasting blood glucose, serum insulin, homeostasis model assessment (HOMA-IR), and liver enzymes were measured. Liver biopsies were evaluated for
\end{abstract}

\footnotetext{
C. Daltro $(\bowtie) \cdot$ L. Boente $\cdot$ R. Leal $\cdot$ T. Portugal

Department of Internal Medicine,

Bahiana School of Medicine and Public Health,

Rua Clarival do Prado Valladares, 264/803,

Salvador, Bahia 41.820-700, Brazil

e-mail: carlahcd@terra.com.br

H. P. Cotrim

Department of Internal Medicine Hepatology,

Bahia Federal University of Medicine,

Salvador, Bahia, Brazil

E. Alves

Obesity Treatment and Surgery Center,

Salvador, Bahia, Brazil

L. A. de Freitas

Department of Pathology, Bahia Federal University of Medicine,

Salvador, Bahia, Brazil

L. Araújo

Department of Internal Medicine,

Bahia Federal University of Medicine,

Salvador, Bahia, Brazil

features of NAFLD including degrees of steatosis, inflammation, cellular ballooning, and fibrosis. NASH was diagnosed in those with steatosis + ballooning or steatosis + fibrosis. The diagnosis of OSA was based on an apnea/hypopnea index (AHI) $\geq 5$ events/hours.

Results OSA was present in $32(80.0 \%)$, NAFLD in 33 $(82.5 \%)$, and NASH in $32(80.0 \%)$ patients. Patients with $\mathrm{AHI} \geq 15 \mathrm{ev} / \mathrm{h}$ had higher serum insulin levels $(30.0 \pm 12.8$ vs. $22.6 \pm 17.3 \mu \mathrm{U} / \mathrm{ml} ; p=0.015)$ and HOMA-IR $(7.5 \pm 4.0$ vs. $5.4 \pm 4.1 ; p=0.016)$ when compared with those with $\mathrm{AHI}<15 \mathrm{ev} / \mathrm{h}$, but no association was found between AHI and NASH $(81.0 \%$ vs. $78.9 \% ; p=1.000)$ or oxihemoglobin desaturation $<84 \%$ and NASH $(81.2 \%$ vs. $70.8 \% ; p=0.709)$ when these groups were compared.

Conclusions Obese patients had elevated OSA and NAFLD frequencies. OSA was associated with insulin resistance but not with the severity of NASH.

Keywords Obesity · Insulin resistance - Oxihemoglobin desaturation $\cdot$ Respiratory sleep disorders $\cdot$ Steatosis

\section{Introduction}

Obstructive sleep apnea (OSA) and nonalcoholic fatty liver disease (NAFLD) are two of many diseases associated with obesity. OSA is a complex disorder consisting of upper airway obstruction, chronic intermittent hypoxia, and sleep fragmentation [1]. Recently studies have shown an association between insulin resistance and OSA. Most of them suggest that OSA can cause insulin resistance, oxidative stress, and cytokine production [2-5], and others propose that obesity and insulin resistance play an important role in the pathogenesis of OSA [6].

Although the pathogenesis of NAFLD is not fully understood, a "two-hit" hypothesis has been proposed [7]. 
The first hit represents the accumulation of triglyceride in hepatocytes secondary to dysregulation of fatty acids and insulin resistance. The second hit, which is thought to result in progression of steatosis to a necroinflammatory hepatitis (nonalcoholic steatohepatitis or NASH), is associated with oxidative stress and cytokine expression.

The association of OSA and NAFLD has been the target of growing attention. OSA could cause not only insulin resistance but worse NAFLD through chronic intermittent hypoxia [2-5, 8]. Several studies in obese patients submitted to bariatric surgery have been conducted, but the results are controversial. The aim of this study was to evaluate the frequency of OSA and NAFLD in obese patients and the relationship between OSA, insulin resistance, and severity of steatohepatitis (NASH).

\section{Materials and Methods}

\section{Study Design}

Consecutive patients undergoing bariatric surgery between March 2007 and December 2008 in a center for surgical obesity were included prospectively in the study. Patient inclusion criteria were body mass index $(\mathrm{BMI})>40 \mathrm{~kg} / \mathrm{m}^{2}$ or $\geq 35$ with comorbidities. Exclusion criteria were known diagnosis of other liver disease than NAFLD, use of continuous positive airway pressure, current use of hepatotoxic drugs, excess alcohol intake (defined as $\geq 20 \mathrm{~g} /$ day or $140 \mathrm{~g} /$ week), and history of HIV disease. All patients gave their informed consent, and the study was approved by the local ethics committee.

\section{Patients}

Demographic and anthropometric characteristics were assessed. The presence or a history of hypertension, diabetes mellitus, or dyslipidemia was also recorded. Laboratory tests included plasma aminotransferase (ALT), aspartate aminotransferases (AST), gamma glutamyltransferase (GGT), alkaline phosphatase, fasting lipid profile, serum glucose level, and serum insulin. Iron studies, and hepatitis B and $\mathrm{C}$ serologies were also performed. The homeostasis model assessment (HOMA-IR) was calculated as fasting serum insulin $(\mu \mathrm{U} / \mathrm{ml}) \times$ fasting blood glucose $(\mathrm{mg} / \mathrm{dl}) \times 0.056 / 22.5$.

All subjects underwent overnight polysomnography, and the reports were issued in accordance with standard criteria [9]. Polysomnography recorded electroencephalogram (electrodes $\mathrm{C} 3, \mathrm{C} 4, \mathrm{O} 1$, and $\mathrm{O} 2$ ), oculogram, electromyogram (electrodes in the mental, submental, and regions of the inferior limbs), electrocardiogram, air flow (thermistor/nasal cannula), respiratory effort (thoracic and abdominal belt), snoring (microphone on the chin), and body position (sensor on the thoracic belt). Oxyhemoglobin saturation was measured by pulse oximetry $\left(\mathrm{SpO}_{2}, \%\right)$. The respiratory events were defined as follows: apnea (air-flow interruption $\geq 10 \mathrm{~s}$ ) and hypopnea (a $50 \%$ or greater reduction in air flow for a period $\geq 10 \mathrm{~s}$ ), accompanied by a $4 \%$ or greater dessaturation and/or a micro-awakening. Mixed apneas were also included in the apnea-hypopnea index (AHI). AHI was obtained dividing the total number of respiratory events by the hours of sleep $(\mathrm{ev} / \mathrm{h})$. OSA was classified in accordance with the AHI as follows: no apnea, fewer than $5.0 \mathrm{ev} / \mathrm{h}$; light apnea, 5.0 and $14.9 \mathrm{ev} / \mathrm{h}$; moderate apnea, 15.0 and $30.0 \mathrm{ev} / \mathrm{h}$; and severe apnea, $>30.0 \mathrm{ev} / \mathrm{h} \mathrm{[1]}$.

Liver biopsy specimens had been obtained intraoperatively during bariatric surgery (needle biopsy). Fresh specimens were fixed in $10 \%$ neutral buffered formalin and stained with hematoxylin-eosin, reticulin, red Picro-sirio, and Perls for iron. Histologic evaluation was performed by a liver pathologist in a blinded fashion. NASH was defined as steatosis plus hepatocytes ballooning degeneration or steatosis plus fibrosis. Steatosis was scored according to the number of affected hepatocytes as: $0(<5 \%), 1(6-25 \%), 2$ (25-50\%), 3 (50-75\%), 4 ( $\geq 75 \%)$.

\section{Data Analysis}

The Statistical Package for Social Science (SPSS Inc., Chicago, IL, USA, Release 16.0.2, 2008) was applied for statistical analysis. Continuous variables were expressed as the mean \pm standard deviation $( \pm \mathrm{SD})$, added to the median (Md) and interquartile range (IQR) when the variable did not have a normal distribution. The variable categories were expressed as percentages and were compared using Chi square or the Fisher exact test. For comparison of the continuous variables between two groups, the Student $t$ test or the Mann-Whitney test was used. Spearman's correlation test was used to study the correlations between the variables. $p$ value $<0.05$ was considered significant.

\section{Results}

\section{Patient's Characteristics}

Of the 40 patients studied, $26(65.0 \%)$ were female. The average age was $36.2 \pm 9.6$ years with an average BMI of $41.6 \pm 4.7 \mathrm{~kg} / \mathrm{m}^{2}$. OSA was diagnosed in $32(80.0 \%)$, NAFLD in $33(82.5 \%)$, and steatohepatitis in $32(80.0 \%)$ of the patients studied (Table 1). Diabetes was diagnosed in five $(12.5 \%)$ patients, and seven $(17.5 \%)$ presented with glycemia between 99 and $125 \mathrm{mg} / \mathrm{dl}$. The average levels of serum insulin were $25.8 \pm 15.8 \mu \mathrm{U} / \mathrm{ml}(\mathrm{Md}=21.1$; IQR 14.9$32.1 \mu \mathrm{U} / \mathrm{ml}$ ), while the HOMA-IR index varied from 2.0 to 18.1 , with an average of $6.3 \pm 4.1(\mathrm{Md}=4.9$; IQR 3.5-7.5) and without any difference based on gender (women 6.0 \pm 4.0 
Table 1 General characteristics of the 40 patients studied, together and separated by gender

The remaining comparisons are not statistically significant. Continuous variables are expressed as the mean \pm standard deviation and categorical variables as a simple and relative frequency

${ }^{\text {a }} p=0.051$

${ }^{\mathrm{b}} p=0.006$

\begin{tabular}{llll}
\hline Variables & Total & Female & Male \\
& $n=40$ & $n=26(65 \%)$ & $32.8 \pm 7.8$ \\
\hline Age (years) & $36.2 \pm 9.6$ & $38.0 \pm 10.2$ & $41.9 \pm 3.6$ \\
BMI $\left(\mathrm{kg} / \mathrm{m}^{2}\right)$ & $41.6 \pm 4.7$ & $41.5 \pm 5.2$ & $126.0 \pm 11.2^{\mathrm{a}}$ \\
Waist circumference (cm) & $120.8 \pm 12.1$ & $117.9 \pm 11.8$ & $44.4 \pm 4.2$ \\
Neck circumference (cm) & $40.6 \pm 4.3$ & $38.1 \pm 1.8$ & $1(7.1 \%)$ \\
Diabetes & $5(12.5 \%)$ & $4(15.4 \%)$ & $7(50.0 \%)$ \\
Dyslipidemia & $21(52.5 \%)$ & $14(53.8 \%)$ & $8(57.1 \%)$ \\
Arterial hypertension & $19(47.5 \%)$ & $11(42.3 \%)$ & $12(85.7 \%)$ \\
OSA (AHI $\geq 5$ ev/h) & $32(80.0 \%)$ & $20(76.9 \%)$ & $1(8.3 \%)$ \\
Light & $16(50.0 \%)$ & $15(75.0 \%)$ & $4(33.3 \%)$ \\
Moderate & $6(18.8 \%)$ & $2(10.0 \%)$ & $7(58.3 \%)$ \\
Severe & $10(31.2 \%)$ & $3(15.0 \%)$ & $29.0(14.1-47.2)^{\mathrm{b}}$ \\
AHI $(\mathrm{Md} ;$ AIQ) & $11.4(6.0-30.0)$ & $9.4(5.2-13.4)$ & 78.6 \\
NAFLD (\%) & 82.5 & 84.6 & 78.6 \\
Steatohepatitis (\%) & 80.0 & 80.8 & 57.1 \\
Elevated ALT $(\%)$ & 22.2 & 12.0 & 14.3 \\
Elevated AST (\%) & 5.0 & 0.0 & 92.9 \\
AST/ALT<1 (\%) & 92.3 & 92.0 & \\
\hline
\end{tabular}

vs. men $6.7 \pm 4.4 ; p=0.606)$. Arterial hypertension and dyslipidemia were present in 19 (47.5\%) and 21 (52.5\%) patients, respectively. Dyslipidemia was distributed as follows: two (9.5\%) hypercholesterolemia, 16 (76.2\%) hypertriglyceridemia, and three (14.3\%) mixed dyslipidemia (Table 1$)$. Only two patients $(5.0 \%)$ smoked. With regards to alcohol consumption, 14 patients $(35 \%)$ consumed $<20 \mathrm{~g} /$ day or $140 \mathrm{~g} /$ week, and 26 (65\%) did not consume alcohol.

\section{Polysomnographic Evaluation}

The average total sleep time was $322.5 \pm 92.7 \mathrm{~min}$. The AHI varied from 1.7 to 98.0, with an average of 11.4 (IQR 6.030.0) ev/h (Table 1). Among the patients with OSA, 16 $(50.0 \%)$ had light, six $(18.8 \%)$ had moderate, and 10 $(31.2 \%)$ had severe OSA. The severe form was predominant among the men (58.3\%), and the light form was predominant among the women $(75.0 \%)$. The average basal oxygen saturation was $98.0 \pm 1.9 \%$, with the medium $\mathrm{O}_{2}$ saturation varying from 94.6 to $98.0 \%$ (a mean of $94.6 \pm$ $1.9 \%$ ) and a minimum $\mathrm{O}_{2}$ saturation varying from 63.0 to $95.0 \%$, with a mean of $82.4 \pm 7.8 \%$. The men presented with a minimum oxygen saturation lower than women, but this difference did not reach statistical significance $(79.4 \pm 9.0 \%$ vs. $84.1 \pm 6.8 \% ; p=0.073)$. The $\mathrm{O}_{2}$ saturation time below $90 \%$ had a median of $0.8 \mathrm{~min}$ (IQR $0.1-11.0$ ).

\section{Liver Evaluation}

The prothrombin/RNI time, total and fractionated proteins, platelets, and total and fractionated bilirubin were normal in all of the patients. The AST and the ALT were higher than the method's upper limit of detection in $5.0 \%$ and $22.2 \%$ of the cases, respectively, and $92.3 \%$ for the patients had AST/ ALT $<1$ (Table 1). Men presented with elevated ALT more frequently than women $(57.1 \%$ vs. $12.0 \% ; p=0.008)$. Table 2 shows the histological findings from the hepatic biopsies of the 40 patients studied. Thirty-three $(82.5 \%)$ of the patients had steatosis with intensity varying from 0 to 4 , being distributed as follows: $0-7(17.5 \%), 1-12(30.0 \%)$,

Table 2 Histological findings from the hepatic biopsies from the 40 obese patients studied

\begin{tabular}{lc}
\hline Histological Findings & $N(\%)$ \\
\hline NAFLD & $33(82.5)$ \\
Steatohepatitis & $32(80.0)$ \\
Steatosis & $33(82.5)$ \\
Macrovesicular & $14(42.4)$ \\
Macro + microvesicular & $19(57.6)$ \\
Inflammation & $32(80.0)$ \\
Lobular & $10(31.2)$ \\
Portal & $3(9.4)$ \\
Lobular + portal & $19(59.4)$ \\
Baloonization & $37(92.5)$ \\
Mallory corpuscles & $4(10.0)$ \\
Fibrosis & $29(72.5)$ \\
Perisinusoidal & $19(65.5)$ \\
Portal & $2(6.9)$ \\
Perisinusoidal + portal & $7(24.1)$ \\
Perisinusoidal + portal + septal & $1(3.4)$ \\
\hline
\end{tabular}


2-13 (32.5\%), 3-3 (7.5\%), and 4-5 (12.5\%). Inflammation was present in $80 \%$ and fibrosis in $72.5 \%$ of cases. Analysis of the histological findings showed no significant differences between genders.

Characteristics of Patients According to Polysomnographic Results

To study the relationship between OSA and the other variables, the patients were divided into two groups according to AHI: $<15$ and $\geq 15 \mathrm{ev} / \mathrm{h}$. We observed that the group with $\mathrm{AHI} \geq 15 \mathrm{ev} / \mathrm{h}$ was predominantly male and had a higher insulinemia and HOMA-IR index when compared with those with $\mathrm{AHI}<15 \mathrm{ev} / \mathrm{h}$ (Table 3). Likewise, the relationship between the oxyhemoglobin desaturation and the other study variables was evaluated by dividing the patients into two groups based on the median of the minimum $\mathrm{O}_{2}$ saturation (84.0\%). In the comparison of the two groups, no statistically significant differences were observed except for AHI (Table 4).

To evaluate the relationship between polysomnographic parameters, insulin resistance, and hepatic enzymes, correlation tests were conducted among these variables. The AHI presented a positive, statistically significant, but weak correlation with HOMA-IR $\left(r_{\mathrm{S}}=0.432 ; p=0.009\right)$ (Fig. 1). In contrast, the correlations between AHI and AST $\left(r_{\mathrm{S}}=\right.$ $0.026 ; p=0.872)$ and AHI and ALT $\left(r_{\mathrm{S}}=0.228 ; p=0.163\right)$ were not statistically significant. The minimum $\mathrm{O}_{2}$ saturation showed a negative and non-statistically significant correlation with HOMA-IR $\left(r_{\mathrm{S}}=-0.292 ; p=0.089\right)$, AST $\left(r_{\mathrm{S}}=-0.018 ; p=0.911\right)$, and ALT $\left(r_{\mathrm{S}}=-0.092 ; p=0.580\right)$. The same occurred between the time of sleep with $\mathrm{O}_{2}$ saturation $<90 \%$ and HOMA-IR $\left(r_{\mathrm{S}}=0.318 ; p=0.066\right)$, ALT $\left(r_{\mathrm{S}}=-0.267 ; p=0.111\right)$, and AST $\left(r_{\mathrm{S}}=-0.138 ; p=0.407\right)$.

\section{Association Between OSA and NAFLD}

To study the relationship between the severity of OSA and oxyhemoglobin desaturation, with NAFLD severity, the histological findings were described according to AHI and the minimum $\mathrm{O}_{2}$ saturation during sleep. As observed in Table 5, there is no association between these variables. The presence of portal inflammation and portal fibrosis also did not show a significant difference between the groups. No significant correlation was found between intensity of steatosis with AHI $\left(r_{\mathrm{S}}=0.181 ; p=0.264\right)$ nor with minimum $\mathrm{O}_{2}$ saturation $\left(r_{\mathrm{S}}=-0.091 ; p=0.576\right)$; however, the intensity of steatosis did show a significant correlation with HOMA-IR $\left(r_{\mathrm{S}}=0.507 ; p=0.002\right)$. The intensity of fibrosis did not correlate with AHI $\left(r_{\mathrm{S}}=0.051\right.$; $p=0.764)$ or with the minimum $\mathrm{O}_{2}$ saturation $\left(r_{\mathrm{S}}=0.002\right.$; $p=0.991)$.

\section{Discussion}

This study demonstrates a high prevalence of OSA and NAFLD among patients with severe obesity that underwent bariatric surgery. We observed a relationship between AHI and insulin resistance, but there is no evidence of an association between AHI or oxyhemoglobin desaturation and the severity of NAFLD.

The pathogenesis and progression of NAFLD have been postulated to occur in two hits. The first constitutes the appearance of hepatic steatosis, as a consequence of the deregulation of fatty acid metabolism and peripheral insulin resistance. The second refers to the progression of steatosis to steatohepatitis due to lipid peroxidation, oxidative stress, and the action of inflammatory cytokines [7].
Table 3 Characteristics of the patients studied according to AHI
Continuous variables are expressed as the mean \pm standard deviation and categorical variables in a simple and relative frequency

\begin{tabular}{llll}
\hline Variables & $\begin{array}{l}\mathrm{AHI}<15 \mathrm{ev} / \mathrm{h} \\
n=24(60 \%)\end{array}$ & $\begin{array}{l}\mathrm{AHI} \geq 15 \mathrm{ev} / \mathrm{h} \\
n=16(40 \%)\end{array}$ & $p$ \\
\hline Gender (\%) & & & 0.016 \\
Female & 87.5 & 31.2 & \\
Male & 12.5 & 68.8 & 0.829 \\
Age (years) & $35.9 \pm 9.0$ & $36.6 \pm 10.9$ & 0.858 \\
BMI (kg/m $\left.{ }^{2}\right)$ & $41.4 \pm 4.5$ & $41.9 \pm 5.1$ & 0.159 \\
Waist circumference $(\mathrm{cm})$ & $118.4 \pm 12.1$ & $124.2 \pm 11.6$ & $<0.001$ \\
AHI (ev/h) & $7.4 \pm 3.8$ & $43.0 \pm 23.8$ & \\
Md (AIQ) & $7.5(3.8-10.7)$ & $33.8(26.8-58.1)$ & 0.610 \\
Glycemia (mg/dl) & $95.8 \pm 10.7$ & $97.8 \pm 12.5$ & 0.015 \\
Insulin ( $\mu$ UU/ml) & $22.6 \pm 17.3$ & $30.0 \pm 12.8$ & 0.016 \\
HOMA-IR & $5.4 \pm 4.1$ & $7.5 \pm 4.0$ & 0.475 \\
Elevated ALT (\%) & 21.7 & 37.5 & 0.162 \\
Elevated AST (\%) & 0.0 & 12.5 & 0.255 \\
AST/ALT<1 (\%) & 87.0 & 100.0 & \\
\hline
\end{tabular}


Table 4 Characteristics of the patients studied according to the oxyhemoglobin desaturation

Continuous variables are expressed as the mean \pm standard deviation and categorical variables in a simple and relative frequency

${ }^{\text {a }}$ Data referring to non-diabetic patients

\begin{tabular}{llll}
\hline Variables & $\mathrm{SatO}_{2} \min <84 \% n=19$ & $\mathrm{SatO}_{2} \min \geq 84 \% n=21$ & $p$ \\
\hline Gender $(\%)$ & & & \\
Female & 57.9 & 71.4 & 0.370 \\
Male & 42.1 & 28.6 & \\
Age (years) & $36.5 \pm 10.6$ & $35.8 \pm 9.0$ & 0.818 \\
BMI $\left(\mathrm{kg} / \mathrm{m}^{2}\right)$ & $43.1 \pm 5.8$ & $40.3 \pm 2.9$ & 0.273 \\
Waist circumference $(\mathrm{cm})$ & $121.4 \pm 13.1$ & $120.4 \pm 11.4$ & 0.809 \\
AHI (ev/h) & $27.3 \pm 23.5$ & $16.5 \pm 22.2$ & 0.033 \\
Md (AIQ) & $18(10.1-36.6)$ & $8.5(3.8-21.0)$ & \\
Glycemia (mg/dl) ${ }^{\mathrm{a}}$ & $97.6 \pm 10.6$ & $95.8 \pm 12.2$ & 0.638 \\
Insulin $(\mu \mathrm{U} / \mathrm{ml})^{\mathrm{a}}$ & $30.8 \pm 20.0$ & $21.6 \pm 9.7$ & 0.260 \\
HOMA-IR & $7.6 \pm 5.3$ & $5.2 \pm 2.4$ & 0.246 \\
Elevated ALT & $31.6 \%$ & $25.0 \%$ & 0.648 \\
Elevated AST & $10.5 \%$ & $0.0 \%$ & 0.231 \\
AST/ALT $<1$ & $94.7 \%$ & $90.0 \%$ & 1.000 \\
\hline
\end{tabular}

There is evidence in the literature that points to OSAS as an independent risk factor for insulin resistance [2-5], although other studies have failed to demonstrate improved insulin resistance with the use of CPAP $[10,11]$ to consolidate the relationship of cause and effect between these two conditions. The mechanisms involved are catecholamine release, due to microarousals, with subsequent activation of the hypothalamic-pituitary-adrenal axis, increasing the production of cortisol (both counterregulators of insulin). In addition, chronic intermittent hypoxia, a characteristic of OSAS, can induce the production of inflammatory cytokines and oxidative stress [3] that not only contributes to the increased insulin resistance but also accelerates the process of liver fibrosis leading to the progression of steatosis to steatohepatitis.

Based on this theory, several studies were conducted, and controversial results have been described. The challenge is to demonstrate that an independent association exists between these two conditions and not a mere coincidence due to

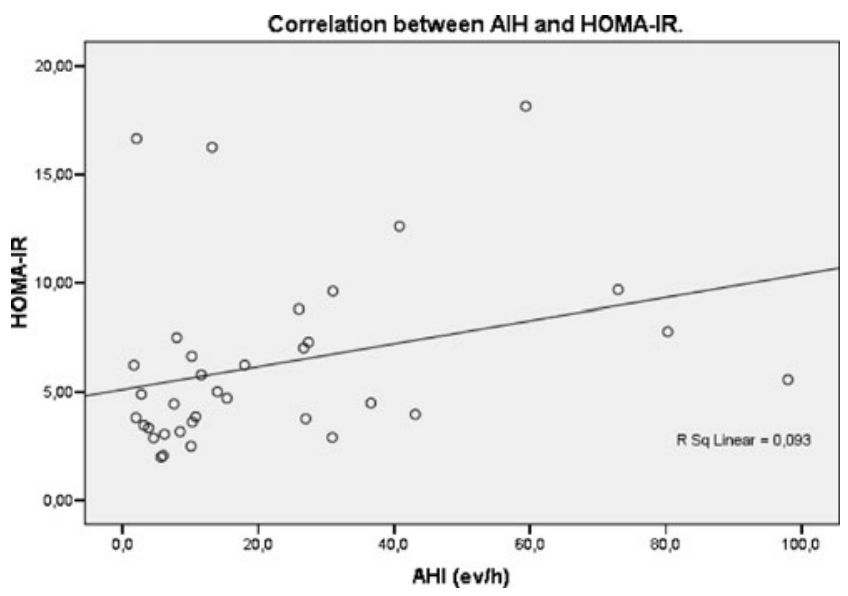

Fig. 1 Correlation between IAH and HOMA-IR having obesity as the common denominator, a risk factor already established for insulin resistance, OSAS and NAFLD [12].

Initially, some authors showed this association in obese and overweight individuals by imaging and liver enzyme exams. Chin et al. [13] observed that treatment with CPAP produced a significant reduction in AST and ALT levels during the first night of treatment. Tatsumi and Saibara [14] studied overweight patients (83 with sleep apnea and 41 controls) using CT for NAFLD diagnosis and measurements of procollagen type III to detect steatohepatitis. They concluded that OSAS was a risk factor for visceral fat and suggested that oxyhemoglobin desaturation during sleep was a risk factor for steatohepatitis, especially in patients with steatosis. However, the principle limitation of these studies concerns the lack of histological data, which does not permit an assessment in concrete terms of the presence and severity of NAFLD.

At the same time, Tanné et al. [15] evaluated 163 patients with suspected OSAS and concluded that those with severe OSAS $(\mathrm{AHI} \geq 50 \mathrm{ev} / \mathrm{h})$ presented with greater insulin resistance, had higher percentage of steatosis, hepatic necrosis. and fibrosis, despite having the same BMI. In this study, the liver enzymes were measured in all of the patients, but the histological data were only obtained from half of the 32 patients with elevated enzyme levels.

The study by Singh et al. [16] was contrary to the data that had been previously published. In this report, 190 patients with a biochemical diagnosis of NAFLD were studied (116 with steatosis and 74 with steatohepatitis), of which 50 had undergone hepatic biopsy, and there was no difference in the prevalence of the symptoms of OSAS among the patients with steatosis and steatohepatitis. It should be noted, however, that this study was limited by the fact that OSAS was assessed through a sleep questionnaire, 
Table 5 Histological findings according to AHI and minimum $\mathrm{O}_{2}$ saturation

\begin{tabular}{|c|c|c|c|c|c|c|}
\hline Histological findings & $\begin{array}{l}\mathrm{AHI}<15 \mathrm{ev} / \mathrm{h} \\
n=24\end{array}$ & $\begin{array}{l}\mathrm{AHI} \geq 15 \mathrm{ev} / \mathrm{h} \\
n=16\end{array}$ & $p$ & $\begin{array}{l}\mathrm{SatO}_{2} \min <84 \% \\
n=19\end{array}$ & $\begin{array}{l}\mathrm{SatO}_{2} \min \geq 84 \% \\
n=21\end{array}$ & $p$ \\
\hline Steatohepatitis & 75.0 & 87.5 & 0.439 & 78.9 & 81.0 & 1.000 \\
\hline Steatosis & 79.2 & 87.5 & 0.681 & 84.2 & 81.0 & 1.000 \\
\hline Inflammation & 79.2 & 81.2 & 1.000 & 78.9 & 81.0 & 1.000 \\
\hline Portal & 63.2 & 76.9 & 0.662 & 86.7 & 52.9 & 0.095 \\
\hline Baloonization & 91.7 & 93.8 & 1.000 & 84.2 & 100.0 & 0.098 \\
\hline Mallory corpuscles & 12.5 & 6.2 & 0.638 & 10.5 & 9.5 & 1.000 \\
\hline Fibrosis & 66.7 & 81.2 & 0.515 & 68.4 & 76.2 & 0.583 \\
\hline Portal & 31.2 & 38.5 & 0.989 & 38.5 & 31.2 & 0.989 \\
\hline
\end{tabular}

Results are expressed as percentages

and the hepatic biopsy was not performed on all of the patients.

While evaluating the association between OSAS with liver enzymes, Kohler et al. [17] published a randomized clinical trial where 94 patients with moderate to severe OSAS were assigned to receive therapeutic or subtherapeutic CPAP for 4 weeks. The serum levels of AST and ALT were compared before and after the use of CPAP between and within groups. Treatment with CPAP showed no beneficial effect on the aminotransferase levels when compared with the subtherapeutic CPAP.

In 2007, Savransky [8] showed (in an animal model) that in the absence of obesity, chronic intermittent hypoxemia could cause hepatic damage through oxidative stress and also could cause an accumulation of glycogen in the hepatocytes, sensitizing the liver to a new insult. At the same time, a series of studies emerged that utilized liver biopsy and polysomnography to evaluate severely obese patients that underwent bariatric surgery, and the present study should be included within this group [18-21]. These studies evaluated the association between OSAS and NAFLD, attempting to show the role of sleep apnea in the two hits that constitute the pathogenesis of NAFLD. Thus, to study the influence of OSAS on the induction of steatosis, it was searched for a relationship between AHI and oxyhemoglobin desaturation with insulin resistance, and to study the influence of sleep apnea on the progression of NAFLD, we evaluated the relationship between AHI and oxyhemoglobin desaturation with histopathological findings indicative of the severity of NAFLD.

In the present study, a high frequency of sleep apnea was demonstrated, which is in agreement with the literature that reports the presence of OSA in approximately $80 \%$ of patients with severe obesity $[15,18,20-22]$. However, comparing the frequency of NAFLD among these works is difficult because Mishra and Younossi [20] only included patients with NAFLD in their study, and Polotsky et al. [21] only performed biopsies on 20 of the 90 obese patients studied. Jouët et al. [18] reported that only five (8.1\%) of the 62 patients evaluated had a normal liver, and NASH was diagnosed in $34.4 \%$ of the patients studied. Furthermore, Kallwitz et al. [19] studied 85 patients and noted that all but one of the patients had NAFLD.

As to these reports' responses to the questions that originally motivated the studies, the results are conflicting. Jouët et al. [18] reported that OSA and the male gender were risk factors for the increase in hepatic enzymes but found no association between AHI and steatosis, fibrosis, and necroinflammatory activity. Regardless, the presence of $\mathrm{NASH}$ was equal in the groups with or without OSA $(\mathrm{AHI} \geq 10 \mathrm{ev} / \mathrm{h})$. The authors did not investigate the association of AHI and oxyhemoglobin desaturation with insulin resistance. Kallwitz et al. [19] concluded, from retrospectively collected data, that there is a tendency for a higher prevalence of OSA (AHI $\geq 15 \mathrm{ev} / \mathrm{h}$ ) in patients with progressive liver disease, indicated by inflammation and fibrosis $(73.3 \%)$, when compared with those that presented with only inflammation of hepatocytes $(45.8 \%)(p=0.06)$. The authors also did not investigate the association of AHI and oxyhemoglobin desaturation with insulin resistance. The study published by Mishra and Younossi [20] presents a greater casuistic and brings discordant results from the previous studies. They noted that patients with NASH had greater AHI, lower levels of oxyhemoglobin desaturation, and minimum $\mathrm{O}_{2}$ saturation during sleep when compared with those who had only hepatic steatosis. The authors suggested that nocturnal hypoxemia as a result of OSA $(\mathrm{AHI}>5 \mathrm{ev} / \mathrm{h})$ may be a risk factor for NASH. In this study, HOMA-IR was evaluated in only a subset of the patients, thus not providing the ability to access the relationship between insulin resistance and OSA in patients with NAFLD. Polotsky et al. [21] studied a sample of severely obese patients, who were rigorously selected, excluding diabetic patients to reduce confounding variables. In the 90 patients included in that study, HOMA-IR and C-reactive protein were researched in addition to the polysomnography, 
but a liver biopsy was only obtained in 20 patients. The results showed that an oxyhemoglobin desaturation greater than $4.6 \%$ was associated with a 1.5 -fold insulin resistance increase. The histopathological data suggested that the nocturnal oxyhemoglobin desaturation may predispose to hepatic inflammation, hepatocyte ballooning, and liver fibrosis but not to steatosis. There was no relationship between the sleep-disordered breathing index and insulin resistance or liver histopathology. In conclusion, the authors state that the stress caused by hypoxia, resulting from sleep apnea, may be implicated in the development of insulin resistance and steatohepatitis in individuals with severe obesity.

Though the data collected here comes from a smaller number of patients, the relationship of OSA with NAFLD in the most complete form was evaluated, observing the role of the AHI and oxyhemoglobin desaturation in all stages of NAFLD. It was found that HOMA-IR was higher in patients with increasingly elevated AHI, but a relationship between oxyhemoglobin desaturation and this indicator of insulin resistance was not observed. A positive association between HOMA-IR and the intensity of steatosis was also observed, suggesting that OSA may contribute to hepatic steatosis by increasing insulin resistance.

No evidence was found for the association of AHI and the oxyhemoglobin desaturation with the severity of NAFLD. When fibrosis occurs in adults with NAFLD, collagen deposition occurs initially in the space of Disse (perissinusoidal space), and with the progression of disease, fibrosis extends into the portal and periportal region [22]. Recently, Brunt et al. [23] published an article suggesting that chronic portal inflammation may be a marker of advanced NAFLD. In this study, no correlation of AHI and oxyhemoglobin desaturation with the intensity of fibrosis was found, and there was also no correlation of portal inflammation and portal fibrosis with polysomnographic parameters.

An analysis of the data, separating men and women, shows that men have on average a greater waist and neck circumference, more frequently elevated ALT, a predominance of severe OSA, and a tendency for a lower minimum $\mathrm{O}_{2}$ saturation when compared with women. However, there is no evidence that the association being studied behaved differently in the two sexes.

The limitations of these last studies can be discussed together because all of their methodologies are similar. The results were obtained only in obese patients, who may not represent the entire population of patients with NAFLD, and obesity in and of itself is a cause of hepatic fibrosis, which leads to a strong confounding variable. All of the studies are observational, lacking prospective character studies to better assess this association, and finally, oxidative stress, resulting from intermittent hypoxia, as a mechanism of action of the progression of NAFLD was not investigated by any of these studies [12].
Faced with what we propose in this discussion, based not only on the results of the current study but also on the literature review, some issues may be raised. Could the results be conflicting due to the different evaluation criteria for OSAS and NAFLD? Does the association exist, but with such a small magnitude that it does not appear in all of the studies? Is this association due to oxyhemoglobin desaturation and not the number of sleep breathing disruptions presented by the patients, which only would become evident in samples of patients whose desaturation was important?

Additionally, another view is worth exploring in the study of this association. OSAS could contribute in augmenting insulin resistance and consequently hepatic steatosis, while the oxidative stress resulting from intermittent hypoxia would make the hepatocytes more vulnerable to the action of other risk factors for the progression of NAFLD [8]. The studies published thus far have shown conflicting results, leaving a gap to be filled with regards to the association addressed here. Given the increasing prevalence of obesity in the world, and considering the high frequency with which these conditions affect the obese, further research is required to clarify the doubts that still exist.

In conclusion, this study showed that obese patients had elevated OSA and NAFLD frequencies. OSA was associated with insulin resistance but not with the severity of NASH. Other studies are necessary to establish whether there is an independent association between OSA and NAFLD or if it is just a coincidence.

Conflict of Interest The authors declare that they have no conflict of interest.

Financial Disclosures The medical student Leonardo Boente received research support from Fundação de Amparo à Pesquisa do Estado da Bahia (FAPESB - BOL0554/2008). The rest of the authors did not receive any funds for this research.

\section{References}

1. American Academy of Sleep Medicine Task Force. Sleep-related breathing disorders in adults: recommendations for syndrome definition and measurement techniques in clinical research. Sleep. 1999;22:667-89.

2. Makino S, Handa H, Suzukawa K, et al. Obstructive sleep apnoea syndrome, plasma adiponectin levels and insulin resistance. Clin Endocrinol. 2006;64:12-9.

3. Vgontzas AN, Papanicolaou DA, Bixler EO, et al. Sleep apnea and daytime sleepiness and fatigue: relation to visceral obesity, insulin resistance, and hypercytokinemia. J Clin Endocrinol Metab. 2000;85:1151-8. 
4. Ip MSM, Lam B, MMT NG, et al. Obstructive sleep apnea is independently associated with insulin resistance. Am J Respir Crit Care Med. 2002;165:670-6.

5. Punjabi NM, Sorkin JD, Katzel LI, et al. Sleep-disordered breathing and insulin resistance in middle-aged and overweight men. Am J Respir Crit Care Med. 2002;165:677-82.

6. Vgontzas AN. Does obesity play a major role in the pathogenesis of sleep apnoea and its associated manifestations via inflammation, visceral adiposity and insulin resistance? Arch Physiol Biochem. 2008;114(4):211-23.

7. Day CP, James OF. Steatohepatitis: a tale of two "hits"? Gastroenterology. 1998;114(4):842-5.

8. Savransky V, Nanayakkara A, Vivero A, et al. Chronic intermittent hypoxia predisposes to liver injury. Hepatology. 2007;45:100713.

9. Rechtschaffen A, Kales A. A manual of standardized terminology, techniques, and scoring system for sleep stages in human subjects. Brain Information Service, UCLA: Los Angeles; 1968.

10. Steiropoulos P, Papanas N, Nena E, et al. A Markers of glycemic control and insulin resistance in non-diabetic patients with obstructive sleep apnea hypopnea syndrome: does adherence to CPAP treatment improve glycemic control? Sleep Med. 2009;10 (8):887-91.

11. Cuhadaroglu C, Utkusavaș A, Oztürk L, et al. Effects of nasal CPAP treatment on insulin resistance, lipid profile, and plasma leptin in sleep apnea. Lung. 2009;187(2):75-81.

12. Daltro C, Araújo L, Cotrim HP. Obstructive sleep apnoea and nonalcoholic fatty liver disease: risk factor or just coincidence? Liver Int. 2008;28(8):1047-9.

13. Chin K, Nakamura T, Takahashi K, et al. Effects of sleep apnea syndrome on serum aminotransferase levels in obese patients. Am J Med. 2003;114:370-6.
14. Tatsumi K, Saibara T. Effects of obstructive sleep apnea syndrome on hepatic steatosis and nonalcoholic steatohepatitis. Hepatol Res. 2005;33:100-4.

15. Tanné F, Gagnadoux F, Chazouillères $\mathrm{O}$, et al. Chronic liver injury during obstructive sleep apnea. Hepatology. 2005;41:1290-6.

16. Singh H, Pollock R, Uhanova J, et al. Symptoms of obstructive sleep apnea in patients with nonalcoholic fatty liver disease. Dig Dis Sci. 2005;50:2338-43.

17. Kohler M, Pepperell JC, Davies RJ, et al. Continuous positive airway pressure and liver enzymes in obstructive sleep apnoea: data from a randomized controlled trial. Respiration. 2009;78 (2):141-6.

18. Jouët P, Sabaté JM, Maillard D, et al. Relationship between obstructive sleep apnea and liver abnormalities in morbidly obese patients: a prospective study. Obes Surg. 2007;17:478-85.

19. Kallwitz ER, Herdegen J, Madura J, et al. Liver enzymes and histology in obese patients with obstructive sleep apnea. J Clin Gastroenterol. 2007;41:918-21.

20. Mishra P, Nugent C, Afendy A, et al. Apneic-hypopneic episodes during obstructive sleep apnea are associated with histologic nonalcoholic steatohepatitis. Liver Int. 2008;28(8):1080-6.

21. Polotsky VY, Patil SP, Savransky V, et al. Obstructive sleep apnea, insulin resistance and steatohepatitis in severe obesity. Am J Respir Crit Care Med. 2009;179(3):228-34.

22. Daltro C, Gregorio PB, Alves E, et al. Prevalence and severity of sleep apnea in a group of morbidly obese patients. Obes Surg. 2007;17(6):809-14.

23. Brunt E, Kleiner DE, Wilson LA, et al. Portal chronic inflammation in Nonalcoholic Fatty Liver Disease (NAFLD): a histologic marker of advanced NAFLD-clinicopathologic correlations from the Nonalcoholic Steatohepatitis Clinical Research Network. Hepatology. 2009;49:809-20. 